\title{
A Linear Approach to Multi-Panel Sensing in Personalized Therapy for Cancer Treatment
}

\author{
Camilla Baj-Rossi, Giovanni De Micheli, Fellow, IEEE, and Sandro Carrara, Member, IEEE
}

\begin{abstract}
In this paper, a new approach for evaluating the performance of a multi-panel biosensor using linear algebra is presented. With a system-level mathematical analysis based on graphs and linear algebra, we formulate a new approach for contributions decoupling in a multi-panel biochip for simultaneous detection of anti-cancer drugs, avoiding redundancy and interaction between enzymes. Experimental results have been used to validate the model.
\end{abstract}

Index Terms-Cytochrome $\mathbf{P 4 5 0}$ biosensor, multiplexed drugs detection, personalized therapy, condition number, limit of detection, linear system.

\section{INTRODUCTION}

$\mathbf{T}$ HE personalization of drug therapies has a significant effect on the quality and cost of healthcare [1]. In order to personalize a pharmacological treatment, it is required to monitor the concentration of drugs or their metabolites directly in blood and to extract some important pharmacokinetics parameters. Then the dose of drug can be individually adjusted according to the patient's pharmacokinetics profile [2], in order to increase the accuracy and efficacy of the treatment and to reduce dangerous adverse drug reactions. Amperometric biosensors provide rapid, accurate and quantitative detection of drugs and metabolites with an inexpensive and simple to use set-up [3], [4].

A promising approach is based on cytochrome $P 450$ enzymes (CYP). CYPs possess multiple substrate recognition sites, therefore they are able to metabolize a wide range of drugs [6]. At the present state-of-the-art there are many examples of CYP-based sensors [7], where typically one probe (enzyme) is employed for the recognition of a single target (the drug). What is still missing is a multi-panel approach where many probes are used to recognize many targets, i.e. involving several CYP isoforms for detecting mixtures of drugs.

Multiple drug detection with CYP is a promising task because many pharmacological therapies are based on the administration of several drugs at the same time, such as for

Manuscript received March 19, 2013; revised May 31, 2013; accepted July 18, 2013. Date of publication July 25, 2013; date of current version October 10, 2013. This work was supported by the SNF Sinergia under Project CRSII2 127547/1, "Innovative Enabling Micro-NanoBio-Technologies for Implantable Systems in Molecular Medicine and Personalized Therapy," and in part by the NanoSys project within the frame of the UE program under Grant ERC-2009-AdG-246810. The associate editor coordinating the review of this paper and approving it for publication was Prof. Krikor B. Ozanyan.

The authors are with EPFL, Swiss Federal Institute of Technology, Lausanne CH-1015, Switzerland (e-mail: camilla.baj-rossi@epfl.ch; giovanni.demicheli@epfl.ch; sandro.carrara@epfl.ch).

Digital Object Identifier 10.1109/JSEN.2013.2274873

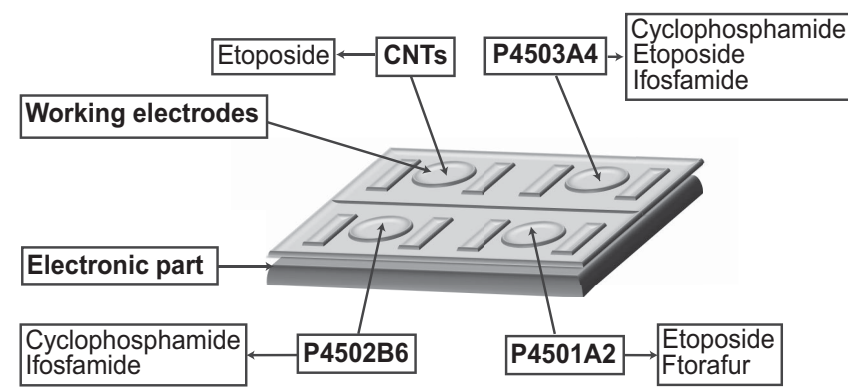

Fig. 1. Multiple-spots sensor scheme. Three working electrodes are nanostructured with multi-walled carbon nanotubes (MWCNTs) and a CYP isoform that is specific for a group of drugs (listed inside the boxes), while one electrode is functionalized with only MWCNTs. MWCNTs are used for enabling drug detection at pharmacological ranges [5].

chemotherapy or cardiovascular diseases [1], [2]. However, a CYP isoform can detect several different compounds at the same time due to the broad substrate range of cytochrome and to its molecular structure [6], not allowing a correct identification and quantification of the drug compound.

Nevertheless in previous works [8], [9], we have shown that by choosing the right combination of CYPs, the simultaneous measurement of two drugs in the same sample is possible, even if it requires complex data analysis. In the development of a cytochrome biosensor array for multiple drug monitoring, proper strategies to distinguish the different drug contributions are therefore necessary to minimize the errors coming from the measurements [10].

The strategy presented in this work is based on linear algebra. In this paper, we propose a system-level mathematical analysis based on a matrix formulation for the decoupling of individual drug contributions in a system containing multiple CYP biosensors. The model, designed to decouple an indefinite number of compounds (in practical clinical cancer, the drugs to be monitored are typically in the range from 1 to 10 ), has been validated with real experimental data, and it can be used for the design of a biosensor array chip for personalized therapy (Fig. 1).

With this paper we want to present the investigation of the possible interactions between the sensors within an array and the strategy that we can adopt to overcome the interferences. In the next sections we will show the mathematical model built on the scheme of the sensor platform reported in Fig. 1, which has been generalized for an undefined number of probes and targets. In Section V we present the validation of the model by a comparison with experimental data. 


\section{Problem Modeling}

In electrochemical biosensors based on CYP we can measure the presence of drugs by analyzing the peaks that are produced in a cyclic voltammogram when the drug bind to the active site of CYP. The presence of a drug induces an increase in the current peak that is proportional to the drug concentration [7].

Since CYPs can host several molecules in their active site, they can detect two different compounds at the same time. With some drug pairs, CYPs will produce multiple peaks in the single cyclic voltammogram [8]. But with other drugs, as reported in [9], we cannot distinguish the contributions of each single drug within a mixture by using only one CYP-sensor, because the current peaks are given at the same potential. The solution to this problem is given by using several CYPs in a sensor array, in the right combination. With this strategy we can decouple the contribution of every drug present in the mixture.

In previous studies many models for avoiding redundancy in sensor applications have been developed: algorithms based on irredundant cover computation, integer linear programming or heuristic models [11], [12]. These models were optimized for different applications: they mainly aim to select a minimum number of probes for the design of arrays, where the input samples may contain any number of probes (which is usually a large number, in case of DNA sensors) [12]. In another study [10], the combinations of sensor spots to be queried were selected by solving a covering problem [13].

The model presented in this paper has the advantage to be based on linear algebra, thus it can be easily implemented to algorithms with low computational costs.

A model based on linear algebra can be applied when sensors give a linear response even in presence of multiple drug interactions. Our previous work [9] shows some examples of detection of drug pairs. In particular we measured the concentration variations of three anti-cancer compounds (Cyclophosphamide, Ifosfamide and Ftorafur) in presence of another compound, Etoposide. As shown in the abovementioned paper, the drug-drug interactions on the same sensor were linear in a certain range of Etoposide concentration (up to $50 \mu \mathrm{M}$ ). This means that for low concentration of Etoposide $(<50 \mu \mathrm{M})$ we can use the linear approximation proposed in this model. And in many clinical applications Etoposide is administered at concentration in the range 1-40 $\mu \mathrm{M}$, as in [14] and in the more recent [15], [16].

However there are some cases where drug-drug interactions are not linear. In the work reported in [8], non linear interactions of several drugs on the same sensors were taken into account. However, that approach is redundant in the case where drug-drug interactions are occurring in a concentration range where they may be sufficiently well modeled by a linear approach.

Here we show how the sensing system can be modeled by a graph and then how it can be analyzed through linear algebra. We introduce a new definition of limit of detection referred to the entire sensor array, which considers all the enzyme spots and the target molecules that enzymes are able

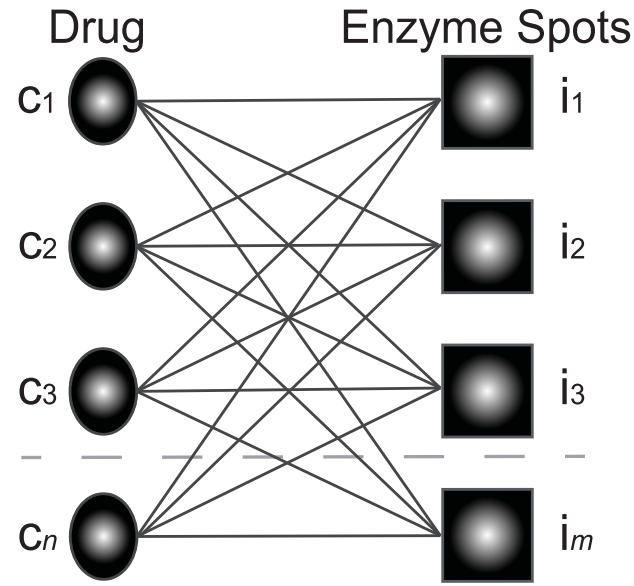

Fig. 2. Graph-scheme: general case with $n$ drug compounds and $m$ enzyme spots. This graph is weighted, i.e. coefficients $s_{l j}$ can be associated with edges and they represent the interaction between drugs and enzymes, i.e. the sensitivity of the $l$-enzyme spot in detecting the $j$-drug (where $1<l<m$; $1<j<n$ ). In general the sensitivity coefficient $s_{l j}>0$ and $s_{l j} \neq 0$, but it may be equal to zero if the $l$-enzyme is not involved in the metabolism of the $j$-drug.

to metabolize. The limit of detection for a single-spot sensor is limited by the measurement and instrument errors, and by the sensor sensitivity [17]. In this new parallel setting, the limit of detection for the entire sensor array $\left(L O D^{*}\right)$, is also affected by limitations due to multiple drug-probe interactions.

In a sensor array, a set of currents is measured, that are indicative of the target concentration. In general

$$
i=f(c)
$$

where $i \in \mathbb{R}^{m}, c \in \mathbb{R}^{n}$ are the current and concentration vectors respectively and $f: \mathbb{R}^{m} \rightarrow \mathbb{R}^{n}$ is the transduction function. All concentrations are of interest within a medical interval, the pharmacological range, $\left[c_{L} c_{M}\right]$, with $c_{L}, c_{M} \in \mathbb{R}^{n}$. If we linearize the function $f$ in the central part of the interval, we can write

$$
S c=i
$$

where $S$ is the Jacobian matrix. We can experimentally show that for the set of sensors that we are studying, the linear approximation is justifiable, since all the performance criteria evaluated for the biosensor, such as the sensitivity and limit of detection, are determined within the linear concentration range of the calibration curve. Electrochemical biosensors always have an upper limit of the linear concentration range. This limit is directly related to the catalytic properties of the biological receptor [18].

\section{GRAPHS AND LineAR SYSTEMS}

The array-based sensor chip reported in Fig. 1 can be abstracted as a bipartite graph (Fig. 2) where two families of vertices are connected together through weighted edges. The first family of vertices (in Fig. 2 labeled as "Drugs"), represents all drug compounds of interest while the second family (the spot column labeled as "Enzyme Spot" in Fig. 2), represents the spots functionalized by different enzymes which are responsible for the catalytic 
reaction enabling the detection mechanism. This graph is edge-weighted and these weights represent the interaction strength between drugs and enzymes. In the general case represented by the graph in Fig. 2, we assume to have $n$ drug spots connected to $m$ enzyme spots. The weight matrix $S=\left\{s_{l j}, l=1,2, \ldots, m ; j=1,2, \ldots, n\right\}$, with $s_{l j} \geq 0$, contains the coefficients of proportionality between the concentration of $j$-drug and the current measured at the $l$-spot, due only to the presence of the drug $j$, thus corresponding to the definition of sensitivity [17] of the single array spot.

$$
\text { Sensitivity }=\frac{\text { Peak Current }}{\text { Drug Concentration }}\left[\frac{n A}{\mu \mathrm{M} \cdot \mathrm{mm}^{2}}\right] \text {. }
$$

The sensitivity coefficient $\mathrm{s}_{l j}$ has a unit of measurement of $\mu \mathrm{A} / \mu \mathrm{M}$ (sometimes it is normalized by the electrode area, e.g. $\mathrm{mm}^{2}$ ), it is always positive and it is equal to zero if the $l$-enzyme is not involved in the metabolism of the $j$-drug. The sensor array operation can be represented as a linear system of $m$ equations in the unknowns represented by vector $c=\left[c_{j}, j=1,2, \ldots ., n\right] \quad[19]$

$$
\left\{\begin{array}{l}
i_{1}=s_{11} c_{2}+s_{12} c_{2}+s_{13} c_{3}+\cdots+s_{1 n} c_{n} \\
i_{2}=s_{21} c_{1}+s_{22} c_{2}+s_{23} c_{3}+\cdots+s_{2 n} c_{n} \\
i_{3}=s_{31} c_{1}+s_{32} c_{2}+s_{33} c_{3}+\cdots+s_{3 n} c_{n} \\
\vdots \\
i_{m}=s_{m 1} c_{1}+s_{m 2} c_{2}+s_{m 3} c_{3}+\cdots+s_{m n} c_{n}
\end{array}\right.
$$

that can be written in the matrix form

$$
\left[\begin{array}{cccc}
s_{11} & s_{12} & \cdots & s_{1 n} \\
s_{21} & s_{22} & \cdots & s_{2 n} \\
\vdots & \vdots & \vdots & \vdots \\
s_{m 1} & s_{m 2} & \cdots & s_{m n}
\end{array}\right]\left[\begin{array}{c}
c_{1} \\
c_{2} \\
\vdots \\
c_{n}
\end{array}\right]=\left[\begin{array}{c}
i_{1} \\
i_{2} \\
\vdots \\
i_{m}
\end{array}\right]
$$

where $i=\left[i_{l}, l=1,2, \ldots ., m\right]$ is the vector of the currents measured with each spot. These are the catalytic currents measured at each $l$-enzyme spot. For the sake of simplicity, starting from this point we assume $m=n$. In an ideal case, each drug is sensed by only one probe. Hence $S$ is diagonal. In reality, the drug-drug interaction makes $S$ a nondiagonal matrix. The contribution of this work is to give a means to assert the accuracy and the limit of detection of the sensor array. Moreover we present a new mathematical approach to decouple the contributions of several drugs, starting from a complex system, where these contributions are coupled.

We assume that our system is consistent and not homogeneous (i.e. we exclude the case where the vector $i$ equals the null vector). In Fig. 3, examples of biosensor structures leading to consistent and inconsistent systems are reported. The coefficient matrix $S$ is chosen to be not singular, i.e. the determinant is not equal to zero, $d(S) \neq 0$. The system does not yield a unique solution if $S$ is not invertible, or equivalently if the rows of $S$ are linearly dependent. We guarantee the system to have a unique solution by choosing in the design phase as many sampling currents as the targets to be measured, and by checking the accuracy of the solution as described in the next section [19].
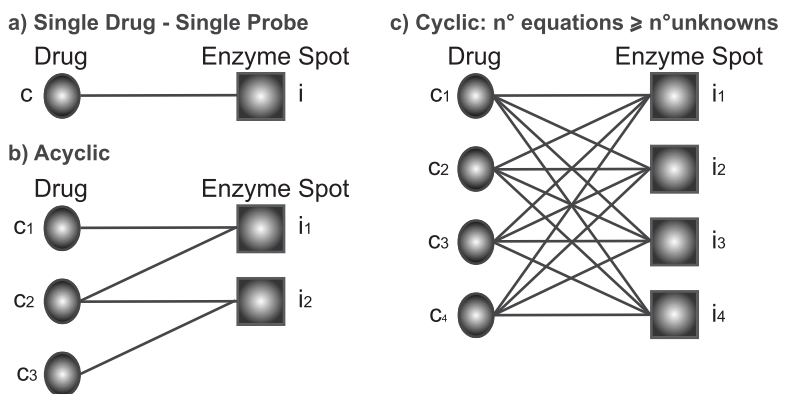

Fig. 3. Examples of consistent and inconsistent systems: in a) and c) the number of equation equals the number of unknowns, thus the systems are consistent, i.e. they have at least one solution (unique or infinite solution); b) is an inconsistent system since the number of unknowns is less than the number of equations.

\section{ACCURACy of Solutions of Linear Systems}

Here we introduce two definitions of error on the concentration. The error on the evaluation of the concentration $\Delta e$ depends on the accuracy of the solution of the linear equation system and it differs from the error on the concentration $\Delta c$, which affects the measurements performed by the sensor. The latter error will be bigger than the previous one since it includes also errors due to the instrument and due to the experimental measurements (random and possible systematic errors).

A system of linear equations is considered to be wellconditioned if a small change in the coefficient matrix or a small change in the right hand side results in a small change in the solution vector [19], [20]. Considering the system in (5), if we change $i+\Delta i$, the new solution $c$ is affected by an error on its evaluation $\Delta e$, defined as

$$
S(c+\triangle e)=i+\triangle i,
$$

where $\Delta i$ is the error on the measured current. Thus the correspondent change in the vector $c$ that is defined as the error on the evaluation of the concentration $(\Delta e)$, is given by

$$
\triangle e=S^{-1} \triangle i .
$$

The perturbations $\Delta e, \Delta i$ and $\Delta S$ are the differences between the measured values of the drug concentration $c_{r}$, the current $i_{r}$ and sensitivity $S_{r}$, and the theoretical values $(c, i$ and $S)$ :

$$
\begin{aligned}
c_{r} & =c+\Delta e \\
i_{r} & =i+\Delta i \\
S_{r} & =S+\triangle S .
\end{aligned}
$$

According to [20] we can assert that the equations system is well-conditioned if small $\Delta i$ results in small $\Delta e$. The solution $c+\Delta e$ is accurate only if the problem of solving $S c=i$ is well conditioned. From a physical standpoint the error $\Delta e$ depends on two factors: 1) the sensitivity of the linear system as represented by $S$ and 2) the error on the transduction as modeled by $\triangle S$. Here we will evaluate the first factor, neglecting the influence of $\triangle S$.

The condition number $\chi(S)$ of $S$ [20]

$$
\chi(S)=\|S\|\left\|S^{-1}\right\|
$$


is the product of the norm of $S$ and the norm of the inverse of $S$. The condition number $\chi(S)$ can quantify the accuracy of the solution of the linear system $S c=i$. It is a function of the norm of a matrix, since the definition of condition number uses the notion of norm to combine information on about a set of numbers into a single number. Thus it is important choosing a norm definition that can properly fit the problem [19]. We can normalize the condition number of $S$ respect to the number of the rows and columns:

$$
\chi(S)=\|S\|\left\|S^{-1}\right\| \frac{1}{N_{m} \cdot N_{m}},
$$

where $N_{m}$ is the number of rows and columns of the matrix $S$. In this way we obtain condition numbers that are independent of the number of electrodes (since with the norm we always consider the maximum values for each row and column of the matrix $S$ ). Let's now independently consider the effects of a perturbation $\triangle i$ on the solution of $S c=i$

$$
S c=i+\triangle i \text {. }
$$

We can demonstrate that the variation of the solution of a nonsingular system $S c=i$ with respect to perturbation $\triangle i$ in $i$ is directly proportional to the condition number of $S$ [19], [20]. From (7) we have

$$
\|\triangle e\| \leq\left\|S^{-1} \triangle i\right\| \leq \frac{\|\triangle i\|}{\|i\|}\left\|S^{-1}\right\|\|i\| .
$$

By computing the relative error in the solution $\varepsilon_{c}=\frac{\|\Delta e\|}{c}$, and knowing that from (2)

$$
\|i\|=\|S c\| \leq\|S\|\|c\|,
$$

we obtain from (9)

$$
\frac{\|\Delta e\|}{\|c\|} \leq \chi(S) \frac{\|\Delta i\|}{\|i\|} \rightarrow \varepsilon_{c} \leq \chi(S) \varepsilon_{i},
$$

which represents an upper limit for $\varepsilon_{c}$. In (14), $\frac{\|\Delta i\|}{\|i\|}$ is the relative error in $i, \varepsilon_{i}$. This means that the relative error in $i$ can be magnified by as much as a factor of $\chi(S)$ in the solution. Inequality (14) asserts that if the condition number of $S$ is small (usually close to unity), the system is wellconditioned, that is the relative error in $i$ is not much larger than the relative error in $c$. If $\chi(S)$ is large $(\chi(S) \gg 1)$, the system is ill-conditioned, that is the relative error in $i$ can be significantly larger than the relative error in $c$.

\section{Model Validation}

Detection limit and sensitivity are the key parameters used for evaluating the sensor quality. The limit of detection $(L O D)$, expressed as a concentration or quantity, is defined as the smallest measure that can be detected with reasonable certainty for a given analytical procedure [17]. For a single sensor of the platform, $L O D$ is calculated as the ratio of the mean of standard deviation current $\triangle i$ (calculated from the background current values), over the analytical sensitivity $S$, [17], [21]

$$
L O D=k \frac{\triangle i}{S}
$$

where $\Delta i$ and $S$ are scalar values. We can introduce a more general definition of limit of detection $\left(L O D^{*}\right)$, for a sensor array with $m$-enzyme spot and $m$-drug compounds to be detected:

$$
L O D^{*}=k\left\|S^{-1} \triangle i\right\|
$$

where $\triangle i, i \in \mathbb{R}^{m}, S \in \mathbb{R}^{m x n}$ and $k$ can have the following values, according to the confidence interval

$$
k=\left\{\begin{array}{l}
1 \rightarrow 68.3 \% \\
2 \rightarrow 95.4 \% \\
3 \rightarrow 99.7 \%
\end{array}\right. \text { confidence interval }
$$

This is a general definition for limit of detection and it can be applied to every array-based sensor with a variable number of enzyme spots and chemicals to be measured. Values of $L O D^{*}$ will be usually larger than the limit of detection evaluated for the single-spot sensor, because, according to the norm definition, with $L O D^{*}$ we always consider the maximum number in the matrix row/column. By introducing in (14) the new definition of $L O D^{*}$, we can obtain an upper bound for the error on the evaluation of the concentration, $\triangle e$ which depends on the condition number and the $L O D^{*}$, according to this inequality

$$
\|\triangle e\| \leq \frac{1}{k} \chi(S) L O D^{*} \frac{\|\Delta i\|\|c\|}{\|\triangle e\|\|i\|}
$$

that can be simplified defining the last term as a generic function $g$ :

$$
\|\triangle e\| \leq \frac{1}{k} \chi(S) L O D^{*} g(\triangle i, \triangle e, i, c) .
$$

This result states that the limit of detection has to be as low as possible because it affects the accuracy of the solution, i.e. if the $L O D^{*}$ is high we estimate the drug concentration with a bad resolution. Similarly, the error on the concentration $\triangle c$ must be at least equal or bigger than the error on the evaluation of the concentration since $\Delta c$ contains also the experimental errors (instrument, measurements, etc.)

$$
\triangle c \geq \triangle e .
$$

We show now the validation of the proposed model on experimental data that were previously published. In our previous work [9], we measured four anti-cancer drugs with four independent sensors in a wet lab. For each sensor we evaluated the sensitivity and limit of detection, as defined in [17]. In order to demonstrate the feasibility of the approach presented in this paper, we evaluate the coefficient matrix $S$ for seven different systems. These systems are thought as if the single sensors tested in [9] were disposed in seven different sensor arrays. Fig. 4 reports all the cases under investigation: in a) the graph for the general case with all four electrodes, in b) the graph for the sensor with three electrodes functionalized with enzymes, in c) all the possible combinations with two drugs, with the corresponding interacting isoforms. For each case we calculated the coefficient matrix $S$ (by taking the sensitivities obtained from experimental data), $L O D^{*}$, and the condition number $\chi(S)$, since these are among the most significant parameters for checking our approach. Table I summarizes the results obtained with the systems illustrated in Fig. 4. Condition numbers and limit of detection (in matrix form), are reported. The values for $L O D^{*}$ are compared with 

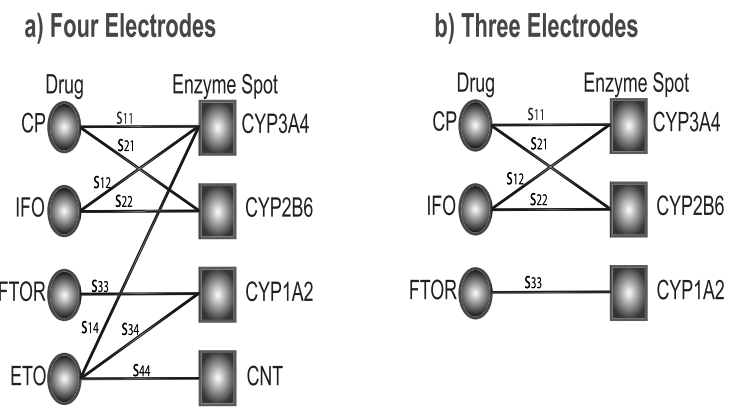
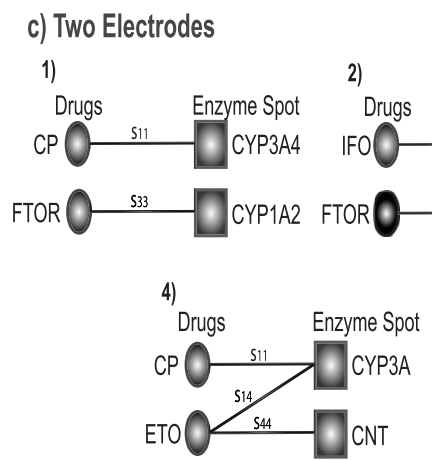
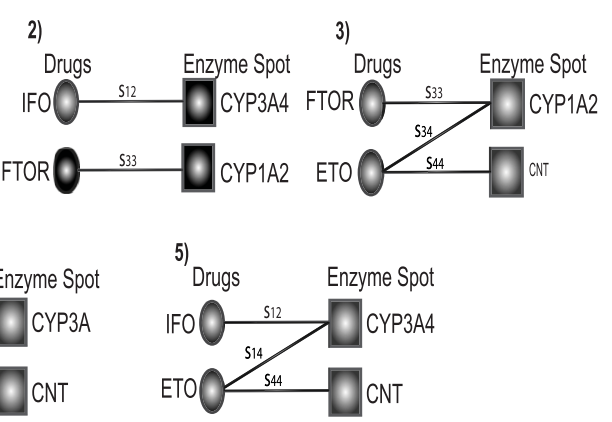

Fig. 4. Graphs of array-based sensor: in a) the graph for the general case with all four electrodes, in b) the graph for the sensor with three electrodes functionalized with enzymes, in c) all the possible combinations with two drugs.

TABLE I

RESULTS FROM THE MODEL AND EXPERIMENTS

\begin{tabular}{cccc}
\hline System & Condition number $\chi(S)$ & $L O D^{*}[\mu M]$ & Measured $L O D[\mu M]$ \\
\hline \hline a) Four electrodes & 14.75 & 7.04 & 4.93 \\
\hline b) Three electrodes & 3.16 & 7.04 & 4.93 \\
\hline c) Two electrodes: & & & \\
\hline 1) CP-FTOR & 2.16 & 4.10 & 2.44 \\
\hline 2) IFO-FTOR & 1.84 & 3.47 & 2.82 \\
\hline 3) FTOR-ETO & 1.39 & 0.56 & 0.69 \\
\hline 4) CP-ETO & 18.06 & 4.10 & 2.44 \\
\hline 5) IFO-ETO & 15.31 & 3.47 & 2.82 \\
\hline
\end{tabular}

the limits of detection obtained by the experimental measurements on each single sensor tested in [9], (Measured LOD in Table I). The LODs* (listed in Table I), that we calculate with our model take into account the possible interferences between enzyme spots. The measured LODs have been obtained with independent sensors. So it is reasonable that LODs* are in general equal or worse than the measured LODs. With two electrodes we obtained LODs* very close to the measured LODs, thus demonstrating: 1) the accuracy of the model exposed previously and 2) that with a sensor array we can obtain more precise measurements than with a single enzyme spot. As expected, for the case with three and four electrodes, the $L O D^{*}$ were larger, but very close to the measured $L O D$. This is due to different reasons: the higher the number of drugs that we want to measure, the more we lose in term of limit of detection, when the off-diagonal terms in the coefficient matrix are significant; according to the definition of $L O D^{*}$, we evaluate the norm of a product (Eq. 16), e.g. we always consider the maximum values for each row and column of the matrix $S$ ). We also obtained fairly good relative error values $\varepsilon_{c}$ : for example, with the two-electrodes chip, we can measure a Cyclophosphamide concentration of $10 \mu \mathrm{M}$ with a detection limit of $4.10 \mu \mathrm{M}$, and with a $2.8 \%$ of error which correspond to $10 \mu \mathrm{M} \pm 0.3 \mu \mathrm{M}$.

\section{CONCLUSION}

In this paper we showed the formal underpinning for designing a sensor array with different enzymes as probe molecules for detection of drugs and chemicals in general. The realized enzyme-array is modeled as a graph and data acquisition has been analyzed by means of linear algebra. We introduced a new definition of limit of detection that is referred to the entire sensor array. The results coming from the validation of the model showed that this model provides a new approach for the automatic detection of multiple drugs with an array of sensors. With this innovative formulation, we can design biosensors with a larger number of probes as well as sensor array with different proteins. We can also integrate other kinds of sensor (such as $\mathrm{pH}$, temperature or glucose sensors), which can further improve the reliability of drug detection. Future works will consider the implementation of this model on a FPGAs or microcontrollers in order to obtain a fully automate array sensor for the simultaneous detection of anti-cancer drugs in therapeutic cocktails.

\section{ACKNOWLEDGMENT}

A. Simalatsar and W. You are acknowledged for their support and useful suggestions on the mathematical model. A. Cavallini is acknowledged for the proofread of the manuscript.

\section{REFERENCES}

[1] S. D. Undevia, G. Gomez-Abuin, and M. J. Ratain, "Pharmacokinetic variability of anticancer agents," Nat. Rev. Cancer, vol. 5, no. 6, pp. 447-458, Jun. 2005.

[2] J. H. Lin, "Pharmacokinetic and pharmacodynamic variability: A daunting challenge in drug therapy," Current Drug Metabolism, vol. 8, no. 2, pp. 109-136, 2007.

[3] J. Wang, "Amperometric biosensors for clinical and therapeutic drug monitoring: A review," J. Pharmaceutical Biomed. Anal., vol. 19, nos. 1-2, pp. 47-53, 1999.

[4] Y. Wang, H. Xu, J. Zhang, and G. Li, "Electrochemical sensors for clinic analysis," Sensors, vol. 8, no. 4, pp. 2043-2081, 2008.

[5] J. J. Gooding, "Nanostructuring electrodes with carbon nanotubes: A review on electrochemistry and applications for sensing," Electrochim. Acta, vol. 50, no. 15, pp. 3049-3060, 2005.

[6] F. P. Guengerich, "Cytochrome p450 and chemical toxicology," Chem. Res. Toxicol., vol. 21, no. 1, pp. 70-83, 2008.

[7] N. Bistolas, U. Wollenberger, C. Jung, and F. W. Scheller, "Cytochrome p450 biosensors-A review," Biosens. Bioelectron., vol. 20, no. 12, pp. 2408-2423, 2005.

[8] S. Carrara, A. Cavallini, V. Erokhin, and G. D. Micheli, "Multi-panel drugs detection in human serum for personalized therapy," Biosens. Bioelectron., vol. 26, no. 9, pp. 3914-3919, 2011.

[9] C. Baj-Rossi, G. D. Micheli, and S. Carrara, "Electrochemical detection of anti-breast-cancer agents in human serum by cytochrome p450-coated carbon nanotubes," Sensors, vol. 12, no. 5, pp. 6520-6537, 2012. 
[10] S. Carrara, A. Cavallini, A. Garg, and G. De Micheli, "Dynamical spot queries to improve specificity in p450s based multi-drugs monitoring," in Proc. ICME Int. Conf. Complex Med. Eng., Apr. 2009, pp. 1-6.

[11] G. W. Klau, S. Rahmann, A. Schliep, M. Vingron, and K. Reinert, "Integer linear programming approaches for non-unique probe selection," Discrete Appl. Math., vol. 155, no. 6, pp. 840-856, 2007.

[12] M. Lombardi, L. Benini, A. Garg, and G. De Micheli, "Methods for designing reliable probe arrays," in Proc. IEEE Int. Conf. BioInformatics BioEng., Jun. 2010, pp. 306-307.

[13] G. De Micheli, Synthesis and Optimization of Digital Circuits, 1st ed. New York, NY, USA: Mcgraw-Hill, 1994.

[14] K. R. Hande, P. J. Wedlund, R. M. Noone, G. R. Wilkinson, F. A. Greco, and S. N. Wolff, "Pharmacokinetics of high-dose etoposide (VP-16213) administered to cancer patients," Cancer Res., vol. 44, no. 1 , pp. 379-382, 1984

[15] R. Patlolla and V. Vobalaboina, "Pharmacokinetics and tissue distribution of etoposide delivered in parenteral emulsion," J. Pharmaceutical Sci., vol. 94, no. 2, pp. 437-445, 2005.

[16] G. Toffoli, G. Corona, R. Sorio, I. Robieux, B. Basso, A. M. Colussi, and M. Boiocchi, "Population pharmacokinetics and pharmacodynamics of oral etoposide," Brit. J. Clinical Pharmacol., vol. 52, no. 5, pp. 511-519, 2001

[17] S. M. J. Mocak, A. M. Bond, and G. Scollary, "A statistical overview of standard (IUPAC and ACS) and new procedures for determining the limits of detection and quantification: Application to voltammetric and stripping techniques (Technical Report)," Pure Appl. Chem., vol. 69, no. 2, pp. 297-328, 1997.

[18] D. R. Thevenot, K. Toth, R. A. Durst, and G. S. Wilson, "Electrochemical biosensors: Recommended definitions and classification," Biosens. Bioelectron., vol. 16, no. 1, pp. 121-131, 2001.

[19] G. W. Stewart, Introduction to Matrix Computations. New York, NY, USA: Academic, 1973.

[20] A. K. Cline, C. B. Moler, G. W. Stewart, and J. H. Wilkinson, "An estimate for the condition number of a matrix," SIAM J. Numer. Anal., vol. 16, no. 2, pp. 368-375, Apr. 1979.

[21] I. Lavagnini, R. Antiochia, and F. Magno, "A calibration-base method for the evaluation of the detection limit of an electrochemical biosensor," Electroanal., vol. 19, no. 11, pp. 1227-1230, 2007.

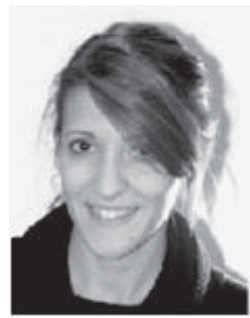

Camilla Baj-Rossi received the M.Sc. degree in biomedical engineering from Politecnico di Torino, Torino, Italy, in 2010.

She carried out research for her master's thesis with École Polytechnique Fédérale de Lausanne (EPFL), Lausanne, Switzerland. She is currently pursuing the Ph.D. degree at the Laboratory of Integrated Systems, EPFL. Her current research interests include the development of a point-of-care biosensor based on multiwalled carbon-nanotubes and cytochrome P450 to detect drugs in biological

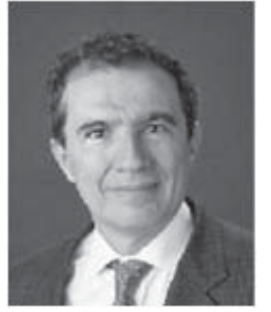

Giovanni De Micheli (F'94) received the Nuclear Engineer degree from Politecnico di Milano, Milano, Italy, in 1979, and the M.Sc. and Ph.D. degrees in electrical engineering and computer science from the University of California, Berkeley, CA, USA, in 1980 and 1983, respectively.

$\mathrm{He}$ is a Professor and the Director of the Institute of Electrical Engineering and the Integrated Systems Center, Ecole Polytechnique Fédérale de Lausanne, Lausanne, Switzerland. He is the Program Leader of the Nano-Terach Program. His current research interests include emerging technologies, networks on chips and 3-D integration, and heterogeneous platform design, including electrical components and biosensors, as well as data processing of biomedical information. He is a fellow of the Association for Computing Machinery and a member of the Academia Europea. He was a recipient of the IEEE Emanuel Piore Award in 2003, the Golden Jubilee Medal from the IEEE CAS Society in 2000, the D. Pederson Award for Best Paper in 1987, two Best Paper Awards from the Design Automation Conference in 1983 and 1993, and the Best Paper Award from the DATE Conference in 2005.

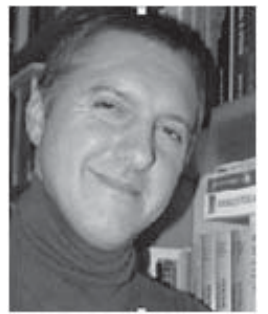

Sandro Carrara (M'04) is a Scientist and Lecturer with École Polytechnique Fédérale de Lausanne, Lausanne, Switzerland. He is a former Professor of optical and electrical biosensors at the Department of Electrical Engineering and Biophysics, University of Genoa, Genoa, Italy, and Former Professor of nanobiotechnology at the University of Bologna, Bologna, Italy.

He is a Founder and Editor-in-Chief of the Journal BioNanoScience (Springer), Topical Editor of the IEEE SENSORS JOURNAL, and an Associate Edito of the IEEE TRANS aCtions on BIOMEDiCAL CiRcuits AND Systems. He is an IEEE member for the Circuit and System Society (CASS) and a member of the Board of Governors of the IEEE Sensors Council. He has been recently appointed as CASS Distinguished Lecturer for 2013 to 2014. His current research interests include electrical phenomena of nano-bio-structured films and complementary metal-oxide-semiconductor design of biochips based on proteins and DNA. He has more than 130 scientific publications and ten patents. He has several Top-25 Hottest-Articles (2004, 2005, 2008, 2009, and two times in 2012) published in highly ranked international journals, such as Biosensors and Bioelectronics, Sensors and Actuators B, IEEE SENSORS JOURNAL, and Thin Solid Films. His work received a NATO Advanced Research Award in 1996 for the original contribution to the physics of singleelectron conductivity in nano-particles, two Best Paper Awards at the IEEE PRIME Conference in 2010 in Berlin and in 2009 in Cork, the Best Poster Award at the Nanotera Workshop in 2011 in Bern, and the Best Poster Award at the NanoEurope Symposium in 2009 in Rapperswil. He received the Best Referees Award from the journal Biosensor and Bioelectronics in 2006. From 1997 to 2000, he was a member of an international committee at the ELETTRA Synchrotron in Trieste. From 2000 to 2003, he was a Scientific Leader of a National Research Program project in nanobiotechnology. He is an internationally esteemed expert on the evaluation panel of the Academy of Finland in a research program for 2010 to 2013. He will be the General Chairman of the conference BioCAS in 2014. 